\title{
HELMINTOS PARASITOS DE MOCÓS (Kerodon rupestris RODENTIA: CAVIIDAE) DE VIDA LIVRE E DE CATIVEIRO, NO SEMI-ÁRIDO NORDESTINO BRASILEIRO
}

\section{Helminth parasites from free-ranging and captive rock cavies (Kerodon rupestris Rodentia: Caviidae) from the semi-arid region of Brazilian northeastern}

\author{
ALMEIDA, K.A. ${ }^{1}$; FREITAS, F.L.C ${ }^{2}$; TEBALDI, J.H. ${ }^{3}$; NASCIMENTO, A.A. ${ }^{3}$ \\ ${ }^{1}$ Universidade Federal do Tocantins \\ ${ }^{2}$ Universidade Federal do Amazonas \\ ${ }^{3}$ Universidade Estadual Paulista - Departamento de Medicina Veterinária Preventiva \\ Endereço para correspondência: Fagner L. C. Freitas - fagner@fcav.unesp.br
}

\section{RESUMO}

Este estudo mostra a fauna helmintológica de 16 mocós (Kerodon rupestris) criados na região semi-árida do Rio Grande do Norte, Brasil. Foram utilizados nove animais capturados no seu habitat natural e sete procedentes de cativeiro localizado no Centro de Multiplicação de Animais Silvestres no município de Mossoró. Após a eutanásia, os animais foram submetidos à necropsia e o trato digestório, traquéia, coração, pulmão, fígado, pâncreas e rins, foram observados quanto à presença de parasitos. Foram identificadas cinco espécies de nematódeos (Paraspidodera uncinata, Trichostrongylus colubriformis, Trichuris gracilis, Trichuris muris e Vianella lenti) e uma de cestódeo (Thysanotaenia congolensis).

Palavras-chave: nematoda, cestoda, roedor, silvestre.

\section{ABSTRACT}

This study shows the helminthic fauna of 16 rock cavies (Kerodon rupestris) from the semi-arid region in the State of Rio Grande do Norte, Brazil. Nine animals were captured from the wild and seven from a captive population located at the Centro de Multiplicação de Animais Silvestres, city of Mossoró, were examined in this study. The animals were slaughtered, and post mortem analysis of the digestive tract, trachea, heart, lungs, liver, pancreas, and kidneys were done in search for the presence of parasites. Five species of nematode (Paraspidodera uncinata, Trichostrongylus colubriformis, Trichuris gracilis, Trichuris muris, and Vianella lenti) and one species of cestode (Thysanotaenia congolensis) were identified.

Key words: nematoda, cestoda, rodent, wildlife.

\section{INTRODUÇÃO}

A espécie Kerodon rupestris, popularmente conhecida como mocó, é um roedor da Família Caviidae, distribuída no Brasil nos
Estados do Nordeste e algumas regiões de Minas Gerais, especificamente em locais rochosos. No Nordeste, é tradicional sua caça e consumo sendo apreciado por sua carne de alta qualidade além do uso da 
pele em artesanato.Esse roedor ainda é pouco estudado, principalmente nos aspectos sanitários. No Brasil, apenas três helmintos foram relatados parasitando a espécie: Strongyloides ferreirai por Rodrigues et al. (1985) e em estudo paleoparasitológico por Araújo et al. (1989); Paraspidodera uncinata (Vicente et al., 1997); e Trichuris sp. (Ferreira et al., 1991; Duarte, 1994).

O gênero Trichuris só foi observado em estudos paleoparasitológicos, sendo encontrados apenas ovos característicos deste gênero. Foram encontrados ovos em coprólitos de 30000 (Ferreira et al., 1991) até 9000 anos antes do presente (AP) (Araújo et al., 1993) no Piauí, e de 2000 anos AP em Pernambuco (Duarte, 1994).

O presente estudo relata os helmintos parasitos de Kerodon rupestris (mocó) de vida livre e de cativeiro, procedentes de Mossoró, Rio Grande do Norte, cidade tipicamente semi-árida da região Nordeste do Brasil.

\section{MATERIAL E MÉTODOS}

Foram utilizados 16 mocós, nove procedentes de vida livre, doados por caçadores (doação das vísceras por residentes rurais que utilizaram a carne para consumo) e sete procedentes do cativeiro, localizado no Centro de Multiplicação de Animais Silvestres, da Escola Superior de Agricultura de Mossoró, Rio Grande do Norte, Brasil.

Os segmentos anatômicos do trato digestório (esôfago, estômago, intestino delgado e intestino grosso), além da traquéia, coração, pulmão, fígado, pâncreas e rins, foram abertos separadamente e o conteúdo tamisado e fixado em solução de Haylliet e Henry. A presença de helmintos foi avaliada em microscópio estereoscópico, sendo então coletados.

Para identificação foram clarificados com ácido acético $80 \%$ e naqueles que necessitaram da observação das formações quitinizadas, utilizou-se posteriormente o creosoto de Faya para uma melhor diafanização. Alguns nematódeos exigiram o estudo da sínlofe, realizado por meio de cortes histológicos transversais. Os cestódeos foram estendidos entre lâminas, imersas em álcool a $70 \%$ durante 30 minutos, no carmin acético por 20 minutos, lavados em álcool a $70 \%, 90 \%$ e $95 \%$, rapidamente, e imersos em creosoto de Faya até obter boa diafanização. Uma vez corados e diafanizados foram montados entre lâmina e lamínula com bálsamo do Canadá.

Os parasitos foram identificados de acordo com Diaz-Ungria (1967), Dronen et al. (1999), Durette-Desset (1968), DuretteDesset (1985), Feliu et al. (2000), Monnig (1947), Pinto et al. (2002), Schmidt (1970), Travassos (1914), Travassos (1921), Vicente et al. (1997).

Utilizou-se a estatística descritiva para determinação dos indicadores de infecção (prevalência, abundância, intensidade e variação de intensidade) de acordo com Margolis et al. (1982).

\section{RESULTADOS}

Todos os 16 animais submetidos à necropsia estavam parasitados, coletandose um total de 113 helmintos, sendo cinco espécies de nematódeos e uma de cestódeo. Estes helmintos estão depositados no Museu de Helmintos de Animais Silvestres do Departamento de Medicina Veterinária Preventiva da Universidade Estadual Paulista, Campus de Jaboticabal. Os nematódeos encontrados foram:

\section{Paraspidodera}

uncinata, caracterizada por apresentar boca com três lábios bem visíveis, esôfago com bulbo na extremidade posterior e um nódulo papiliforme na região posterior do corpo. $\mathrm{O}$ comprimento total das fêmeas variou de 14,12 a $19,79 \mathrm{~mm}$ e o ovo de 0,026 a 0,028 $\mathrm{mm} \times 0,042$ a $0,045 \mathrm{~mm}$. O macho $(12,65 \mathrm{a}$ $14,41 \mathrm{~mm})$ possui ventosa circular de 
rebordo quitinoso $(0,093$ a $0,12 \mathrm{~mm})$, dois espículos subiguais (o menor variando de 0,71 a $0,73 \mathrm{~mm}$ e, o maior de 0,74 a 0,75 $\mathrm{mm}$ ) e vinte e um pares de papilas.

Trichostrongylus colubriformis, caracterizado por ser um helminto pequeno, delgado e de corpo gradualmente atenuado na extremidade anterior. A fêmea apresenta comprimento total de 3,97 a 4,01 $\mathrm{mm}$, útero e ovários pouco sinuosos, ovejetor bem desenvolvido, a vulva na metade posterior do corpo e a extremidade posterior cônica e pontuda. O macho $(3,21$ a $3,32 \mathrm{~mm}$ ) possui espículos iguais e robustos $(0,11$ a $0,14 \mathrm{~mm})$, de coloração castanha, que terminam com um processo triangular, lembrando a ponta de um arpão. Presença de gubernáculo de forma navicular.

Trichuris gracilis, dessa espécie de nematódeo foram encontradas apenas fêmeas com comprimento total de 22,94 a $44,12 \mathrm{~mm}$ e com o ovo medindo 0,060 a $0,066 \mathrm{~mm} \times 0,030$ a $0,034 \mathrm{~mm}$. Caracteriza-se pelo término da banda bacilar na união entre o esôfago e o intestino, sendo a vulva ligeiramente posterior ao ponto de união desses órgãos, e o útero está conectado ao ovejetor por um estreito canal cilíndrico.

Trichuris muris, nematódeo com tamanho que varia de 26,53 a $44,12 \mathrm{~mm}$ de comprimento, com relação anteriorposterior do corpo de 8/5, tamanho do ovo de 0,063 a $0,069 \mathrm{~mm} \times 0,036$ a $0,040 \mathrm{~mm}$, e a fêmea apresenta um ovejetor muscular bem característico.

Vianella lenti, apresenta coloração avermelhada, fortemente espiralada, com dilatação cefálica $(0,04$ a $0,08 \quad \mathrm{~mm})$ separada do corpo por um sulco anelar. A sínlofe se apresenta com eixo de orientação ligeiramente oblíquo, tendendo a frontal; presença de sete cristas longitudinais dirigidas perpendicularmente do lado direito para o esquerdo, sendo quatro cristas ventrais e três dorsais. A fêmea $(3,49$ a $3,52 \mathrm{~mm})$ possui cauda curta e cônica e uma estrutura entre a trompa e o útero que, quando plena, mostra um entumescimento pós-vulvar, tendo o ovo variação de 0,04 a 0,05 $\mathrm{mm} \times 0,03 \mathrm{~mm}$. 0 macho (3,1 a 3,2 mm) apresenta papilas pré-bursais bem visíveis, espículos subiguais $(0,12$ a $0,14 \mathrm{~mm}$ e 0,14 a 0,16 $\mathrm{mm})$ de estrutura complexa e com a extremidade anterior arrendondada.

Thysanotaenia congolensis, anoplocefalídeo de comprimento total de $118,35 \mathrm{~mm}$, com proglótes mais largas que longas, quando maduras, apresentam comprimento de 0,24 a $0,34 \mathrm{~mm}$ e largura de 1,47 a $1,76 \mathrm{~mm}$ e, quando grávidas, de 1,68 a $1,85 \mathrm{~mm}$ e de 1,79 a $1,97 \mathrm{~mm}$, respectivamente. Escólex de 0,31 a 0,34 $\mathrm{mm}$ de comprimento e 0,29 a 0,32 $\mathrm{mm}$ de largura. Poros genitais unilaterais e préequatoriais. Bolsa do cirro piriforme medindo 0,15 a $0,16 \mathrm{~mm}$. Testículos laterais e posteriores ao ovário. Glândula vitelina compacta, pós-ovariana, e a vagina abrindo posteriormente ao cirro. Presença de cápsulas ovígeras $(0,18$ a $0,22 \mathrm{~mm} \times$ $0,16$ a $0,19 \mathrm{~mm})$ com vários ovos $(0,048$ $\mathrm{mm})$.

A tabela 1 apresenta os indicadores de infecções naturais por helmintos observados.

\section{Prevalência das espécies}

T. gracilis foi o helminto com maior prevalência $(71,4 \%)$ nos animais de cativeiro, que também estavam parasitados por $P$. uncinata e $T$. muris, com prevalências de $28,6 \%$ e de $14,3 \%$, respectivamente. Nos animais de vida livre o helminto mais prevalente foi $T$. colubriformis, com $55,6 \%$ dos mocós infectados, seguido por $P$. uncinata $(33,3 \%), \quad V$. lenti $(33,3 \%), \quad T$. gracilis $(22,2 \%), T$. muris $(22,2 \%)$ e $T$. congolensis $(22,2 \%)$.

\section{Abundância}

Nos animais de vida livre, $T$. colubriformis apresentou uma abundância de 6,$33 ; V$. lenti de 1,$56 ; P$. uncinata de 1,$11 ; T$. gracilis de 0,$78 ; T$. congolensis de 
Tabela 1- Indicadores de infecções naturais por helmintos observados em animais da espécie Kerodon rupestris de vida livre $(n=9)$ e de cativeiro $(n=7)$ procedentes do município de Mossoró-RN, Brasil. Indicadores de Infecção

\begin{tabular}{|c|c|c|c|c|c|c|c|c|}
\hline & \multicolumn{2}{|c|}{ Prevalência (\%) } & \multicolumn{2}{|c|}{ Abundância } & \multicolumn{2}{|c|}{$\begin{array}{c}\text { Intensidade } \\
\text { média } / \mathrm{n}^{\circ} \mathrm{de} \\
\text { positivos }\end{array}$} & \multicolumn{2}{|c|}{$\begin{array}{l}\text { Variação de } \\
\text { Intensidade }\end{array}$} \\
\hline & $\begin{array}{l}\text { Vida } \\
\text { Livre }\end{array}$ & Cativeiro & $\begin{array}{l}\text { Vida } \\
\text { Livre }\end{array}$ & Cativeiro & $\begin{array}{l}\text { Vida } \\
\text { Livre }\end{array}$ & Cativeiro & $\begin{array}{l}\text { Vida } \\
\text { Livre }\end{array}$ & Cativeiro \\
\hline Nematódeos & & & & & & & & \\
\hline Paraspidodera uncinata & 33,3 & 28,6 & 1,11 & 0,29 & $3,33(3)$ & $1,0(2)$ & $1-6$ & 1 \\
\hline Trichostrongylus colubriformis & 55,6 & - & 6,33 & - & $11,4(5)$ & - & $1-23$ & - \\
\hline Trichuris gracilis & 22,2 & 71,4 & 0,78 & 1,57 & $3,50(2)$ & $2,2(5)$ & $2-5$ & $1-4$ \\
\hline Trichuris muris & 22,2 & 14,3 & 0,56 & 0,14 & $2,50(2)$ & $1,0(1)$ & $2-3$ & 1 \\
\hline Vianella lenti & 33,3 & - & 1,56 & - & 4,67 (3) & - & $1-11$ & - \\
\hline \multicolumn{9}{|l|}{ Cestódeos } \\
\hline Thysanotaenia congolensis & 22,2 & - & 0,67 & - & $3,00(2)$ & - & 3 & - \\
\hline
\end{tabular}

0,67 e T. muris de 0,56. A abundância foi menor nos animais de cativeiro, sendo $T$. gracilis o mais abundante $(1,57)$ seguido pela $P$. uncinata $(0,29)$ e pelo $T$. muris $(0,14)$.

\section{Intensidade}

A intensidade dos helmintos nos animais de cativeiro foi de um helminto por mocó para $P$. uncinata e para $T$. muris; e para $T$. gracilis de 2,2. Nos animais de vida livre encontrou-se a maior intensidade para $T$. colubriformis, de 11,4 ; seguido por $V$. lenti com 4,67; $T$. gracilis com 3,$5 ; P$. uncinata com 3,33; T. congolensis com 3 e T. muris com 2,5.

\section{Variação de intensidade}

A variação de intensidade para $T$. gracilis, nos animais de cativeiro, foi de 1 a 4 helmintos, não havendo variação para $P$. uncinata e para $T$. muris. Para os animais de vida livre, a variação de intensidade foi de 1 a 6,1 a 23, 2 a 5, 2 a 3, 1 a 11, e de 3 para $P$. uncinata, $T$. colubriformis, $T$. gracilis, $T$. muris, $V$. lenti e $T$. congolensis, respectivamente.

\section{DISCUSSÃO}

A estrutura da fauna helmíntica diferiu da citada no catálogo de Vicente et al. (1997), que descreveram $P$. uncinata e $S$. ferreirai parasitando o mocó. No presente estudo, apenas $P$. uncinata foi encontrada, sendo descritos ainda $T$. colubriformis, T. gracilis, T. muris, V. lenti e $T$. congolensis, pela primeira vez nesse roedor. Ressalta-se que o gênero Trichuris foi considerado extinto nesse hospedeiro (Araújo et al., 1993).

Neste estudo, $P$. uncinata apresentou uma prevalência de $31,3 \%$ e variação de intensidade de 1 a 6 helmintos, valores próximos aos encontrados por Dittmar (2002) quando trabalhou com Cavia aperea (Caviidae), procedentes do Peru, nas quais encontrou uma prevalência de $37 \%$ e variação de intensidade de 0 a 7 helmintos. Já Pinto et al. (2002), pesquisando os helmintos de Cavia porcellus, no Brasil, em dois grupos (cativeiro e "loja de animais"), verificou prevalência, variação de intensidade e intensidade média de 10\%, 1 a 4 e 0,2, respectivamente, para os animais de cativeiro; e de 40\%, 1 a 37 e 5,3, respectivamente, para os animais provenientes de "lojas de animais". Esses 
resultados demonstram que $P$. uncinata é parasito freqüente dentre as espécies de caviídeos.

T. colubriformis é parasito de ruminantes domésticos, suínos, bubalinos e diagnosticado eventualmente no homem (Vicente et al., 1997). Em infecção natural de roedores, esse é o primeiro relato, sendo encontrado no presente estudo apenas nos mocós de vida livre, em razão da convivência no mesmo ecossistema entre estes e as espécies domésticas como bovinos, ovinos e caprinos criados extensivamente.

A presença do gênero Trichuris no mocó, nos dias atuais, é de suma importância em razão dos ovos desse parasito só terem sido encontrados em material arqueológico de Kerodon rupestris até 2000 anos AP (Araújo et al., 1993; Duarte, 1994; Ferreira et al., 1991) e, desde então, não mais encontrados, sugerindo o desaparecimento deste gênero devido as alterações climáticas ocorridas na região Nordeste há 10000 anos, as quais originaram a formação semi-árida existente na atualidade. Posteriormente, em Pernambuco, foram diagnosticados ovos de Trichuris sp., datados de 2000 anos, permitindo aventar a possibilidade de manutenção do ciclo biológico naquela região, onde as condições climáticas, em contraste com outras regiões, não sofreram alterações tão intensas, constituindo paisagem de exceção dentro do domínio semi-árido do Nordeste. Discutia-se, porém, a questão sobre a manutenção da relação entre o parasito, seu hospedeiro e o ecossistema da região nos dias atuais (Duarte et al., 2002). No presente estudo observou-se a presença de duas espécies de Trichuris, mostrando que a relação parasito-hospedeiro não foi afetada pelas alterações climáticas ocorridas na região.

Ferreira et al. (1991) afirmam que a única espécie de Trichuris parasito de roedor que habita o mesmo local dos mocós é o $T$. gracilis, parasito de cutia (Dasyprocta spp.). No entanto descartaram a possibilidade de ser esse helminto, porque as medidas dos ovos (50 a $59 \times 23$ a $28 \mu \mathrm{m})$ não correspondiam às encontradas nos coprólitos (60 a $65 \times 30$ a $33 \mu \mathrm{m}$ ); lembrando que os ovos do gênero Trichuris não são alterados pelas técnicas de dissecação e reidratação utilizada na paleoparasitologia. Porém, um dos Trichuris sp. encontrado neste estudo, $T$. gracilis, apresenta o tamanho dos ovos (60 a $66 \mu \mathrm{m} \times 30$ a $34 \mu \mathrm{m}$ ) semelhantes aos encontrados nos coprólitos dos mocós por Araújo et al. (1989); Ferreira et al. (1991) e Araújo et al. (1993), observando apenas que houve uma variação no tamanho do ovo com a mudança do hospedeiro, pois as demais características são condizentes com essa espécie de parasito; logo sugere ser $T$. gracilis a espécie de Trichuris que está presente no mocó há, pelo menos, 30.000 anos AP e que permanece até os dias atuais.

Essa é a primeira descrição de $T$. gracilis parasitando caviídeos no Brasil, tendo sido encontrado anteriormente somente em Cavia aperea, no Peru (Dittmar, 2002). Deste helminto só foram encontradas fêmeas, achado semelhante aos de Rudolphi (1819 apud Diaz-Ungria, 1967), Cameron e Reesal (1951 apud DiazUngria, 1967) e de Diaz-Ungria (1967) com outras espécies animais. As características da fêmea são suficientes para classificar a espécie.

Já T. muris foi reportado em 18 gêneros de hospedeiros, sendo sete da Ordem Rodentia, portanto considerado um parasito cosmopolita de roedores (Feliu et al., 2000), porém, na Família Caviidae, essa é a primeira descrição.

$O$ parasitismo por $V$. lenti no mocó foi aqui descrito pela primeira vez, ressaltando que apenas os animais de vida livre estavam parasitados, em função do contato com o preá (Galea spixii), hospedeiro habitual desse parasito (Durette-Desset, 1968).

O cestódeo $T$. congolensis é parasito de Thryonomys gregorianus 
(Thrynomyidae) (Dronen et al., 1999), conhecido por rato-da-cana, espécie não encontrada no Brasil. A presença desse parasito em Kerodon rupestris, pela primeira vez no Brasil, deve-se provavelmente às famílias desses roedores terem origem comum (Chame, 2003).

Em conclusão, foram identificadas cinco espécies de nematódeos (Paraspidodera uncinata, Trichostrongylus colubriformis, Trichuris gracilis, Trichuris muris e Vianella lenti) e uma de cestódeo (Thysanotaenia congolensis). O conhecimento dessas espécies de parasitos do mocó vem contribuir com o diagnóstico das helmintoses, bem como, coletar subsídios para melhor controle e, auxiliar no desenvolvimento desse roedor na região semi-árida nordestina.

\section{REFERÊNCIAS}

ARAÚJO, A.; FERREIRA, L. F.; CONFALONIERI, U.; CHAME, M.; RIBEIRO, B. Strongyloides ferreirai Rodrigues, Vicente \& Gomes, 1985 (Nematoda, Rhabdiasoidea) in rodents coprolites (8.000 - 2.000 years BP), from archaelogical sites from Piauí, Brazil. Memórias do Instituto Oswaldo Cruz, v.84, n.4, p.493-496, 1989.

ARAÚJO, A.; RANGEL, A.; FERREIRA, L. F. Climatic change in northeasten Brazil Paleoparasitological data. Memórias do Instituto Oswaldo Cruz, v.88, n.4, p.577-579, 1993.

CHAME, M. Terrestrial mammal feces: a morphometric summary and description. Memórias do Instituto Oswaldo Cruz, v.98, n.1, p.71-94, 2003.

DIAZ-UNGRIA, C. Nematodes parasitos de roedores venezolanos. Sociedad Venezolana de Ciencias Naturales, v.34, n.1, p.129-162, 1967.

DITTMAR, K. Arthropod and helminth parasites of the wild guinea pig, Cavia aperea, from the Andes and the Cordillera in Peru, South America. Journal of Parasitology, v.88, n.2, p.411-414, 2002.

DRONEN, N. O.; SIMCIK, S. R.; SCHARNINGHAUSEN, J. J.; PITTS, R. M. Thysanotaenia congolensis n. sp. (Cestoda: Anoplocephalidae) in the lesser Savanna cane rat, Thryonomys gregorianus from Democratic Republic of Congo, Africa. Journal of Parasitology, v.85, n.1, p.90-92, 1999.
DUARTE, A. N. Estudo paleoparasitológico em coprólitos do sítio arqueológico da Furna do Estrago, Município do Brejo da Madre de Deus, Pernambuco-Brasil. 1994. Rio de Janeiro, $138 f$. Dissertação (Mestrado em Ciências Veterinárias) Curso de Pós-graduação em Ciências Veterinárias, Universidade Federal Rural do Rio de Janeiro.

DUARTE, A. N.; ARAÚJO, A. J. G.; SILVA, L. F. R. F. Paleoparasitologia e Paleoepidemiologia. In: MEDRONHO, R. A. Epidemiologia. São Paulo, Atheneu, 2002, Cap.23, p. 457-463.

DURETTE-DESSET, M. C. Nématodes Héligmosomes d'Amérique du Sud. I. Description de deux nouvelles espèces: Stilestrongylus freitasi, parasite de Zygodontomys lasiurus, et Vianella lenti, parasite de Galea spixi. Bulletin du Muséum National d'Histoire Naturelle, v.40, n.2, p.403-412, 1968.

DURETTE-DESSET, M. C. Trichostrongyloid nematodes and their vertebrate hosts: Reconstruction of the phylogeny of a parasitic group. Advances in Parasitology, v.24, n.1, p.239-305, 1985.

FELIU, C.; SPAKULOVÁ, M.; CASANOVA, J. C.; RENAUD, F.; MORAND, S.; HUGOT, J. P.; SANTALLA, F.; DURAND, P. Genetic and morphological heterogeneity in small rodent whipworms in southwestern Europe: Characterization of Trichuris muris and description of Trichuris arvicolae n. sp. (Nematoda: Trichuridae). Journal of Parasitology, v.86, n.3, p.442-449, 2000.

FERREIRA, L. F.; ARAÚJO, A.; CONFALONIERI, U.; CHAME, M.; GOMES, D. C. Trichuris eggs in animal cropolites dated from 30.000 years ago. Journal of Parasitology, v.77, n.3, p.491-493, 1991.

MARGOLIS, L.; ESCH, G. W.; HOLMES, J. C.; KURIS, A. M.; SCHAD, G. A. The use of ecological terms in parasitology (Report of an ad hoc commite of the American Society of Parasitologists). Journal of Parasitology, v.68, n.1, p.131-133, 1982.

MONNIG, H. O. 1947. Helmintología y entomología veterinarias. Barcelona, Labor, 1947, $434 p$.

PINTO, R. M.; GOMES, D. C.; PEREIRA-MUNIZ, L. C.;NORONHA, D. Helminths of the guinea pig, Cavia porcellus (Linnaeus), in Brazil. Revista Brasileira de Zoologia, v.19, n.1, p.261-269, 2002.

RODRIGUES, H. O.; VICENTE, J. J.; GOMES, D. C. Strongyloides ferreirai sp. n. (Nematoda: Rhabdiasoidea) parasito do roedor Kerodon rupestris (Wied.) no Brasil. Memórias do Instituto Oswaldo Cruz, v.80, n.4, p.407-410, 1985. 
SCHMIDT, G. D. The tapeworms. Colorado, WM. C. Brown, 1970, 266p.

TRAVASSOS, L. Contribuição para o estudo da fauna helmintológica brazileira. Novo gênero da família Heterakidae Railliet \& Henry. Memórias do Instituto Oswaldo Cruz, v.6, n.1, p.137-142, 1914.

TRAVASSOS, L. Contributions à l'étude de la fauna helminthologique du Brézil. XIII. Essai monographique sur la famille des Trichostrongylidae Leiper, 1909. Memórias do Instituto Oswaldo Cruz, v.13, n.1, p.1-135, 1921.

VICENTE, J. J.; RODRIGUES, H. O.; GOMES, D. C.; PINTO, R. M. Nematóides do Brasil. Parte V: Nematóides de mamíferos. Revista Brasileira de Biologia, v.14, n.1, 1977, 452p. 